\title{
Commentary
}

\section{Commentary on A. Habib and M. Rupp (2012), "Antenna Selection in Polarized Multiple Input Multiple Output Transmissions with Mutual Coupling"}

\author{
Rimvydas Aleksiejunas \\ Telecommunications Research Center, Department of Radiophysics, Vilnius University, Sauletekio 9, bldg. III, \\ LT-10222 Vilnius, Lithuania \\ E-mail: rimvydas.aleksiejunas@ff.vu.lt
}

Habib and Rupp [3] provided analytical results for mutual coupling between multiple dipole antennas with angular or spatial separation. However, the method of deriving mutual coupling from mutual impedance involved several inconsistencies on which depend the final results of multiple antenna system capacity presented in their paper.

First, the expression of mutual impedance matrix (12) in [3] is adapted from [1] and contains the sum of two impedances, $Z_{A}+Z_{T}$, on the diagonal. This was a mistake in [1] which was later corrected in [2].

Second, the imaginary part of mutual impedance between skew dipoles shown in Fig. 3 [3] is incorrect and related statement in the text of Section 2.3 saying that "the imaginary part remains basically zero" is not true.

The imaginary part of mutual impedance depends on the angle between dipoles and for the zero angle should coincide with the self-impedance, which is $Z_{11}=$ $73.1+j 42.5 \Omega$ for a half-wavelength dipole. This can be verified by references [4-7], the graphical representation of which results is shown in Fig. 1 illustrating the difference of imaginary part of mutual impedance from zero value.

The equations for mutual impedance (13)-(18) in [3] are based on [677], but have distance $r$ dependent factor $\exp (j k r) / r$ replaced by the factor $\exp (j k x) / x$ with

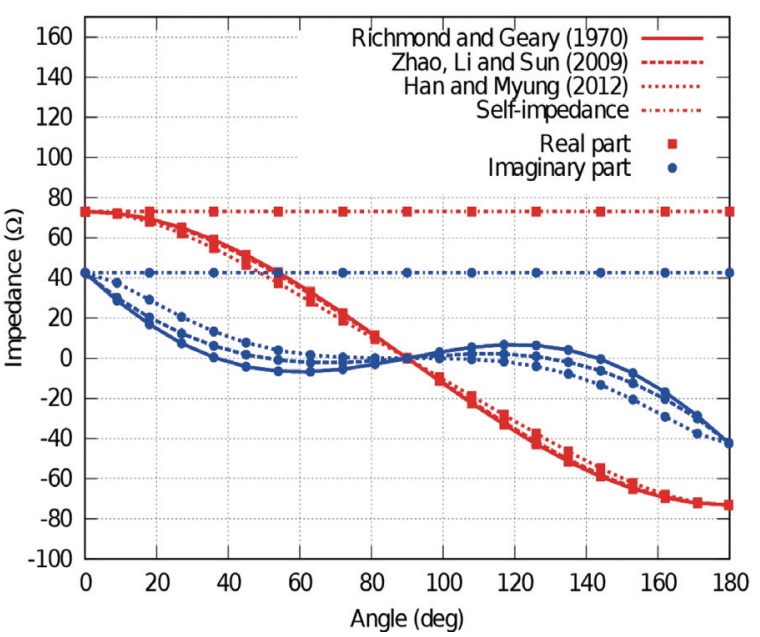

Fig. 1. Dependence of real and imaginary parts of mutual impedance on the angle between skew dipoles according to Richmond and Geary [5], Zhao, Li and Sun [6]7] and Han and Myung [4].

$x$ being integration variable over the length of the dipole. Variable $x$ may have negative and positives values while $r$ is related to $x$ by module, $r=|x|$. This change was not justified and it makes difference for the resultant imaginary part of mutual impedance between dipoles. 
The statement in the title of Fig. 4 [3] that mutual impedance of spatially separated dipoles shown in the figure was obtained from Eq. (18) is inaccurate since that equation was derived only for co-located dipoles.

\section{References}

[1] S. Durrani and M.E. Bialkowski, Effect of mutual coupling on the interference rejection capabilities of linear and circular arrays in CDMA systems, IEEE Transactions on Antennas and Propagation 52(4) (2004), 1130-1134.

[2] S. Durrani and M.E. Bialkowski, Corrections to "Effect of mutual coupling on the interference rejection capabilities of linear and circular arrays in CDMA systems", IEEE Transactions on Antennas and Propagation 53(5) (2005), 1863-1863.
[3] A. Habib and M. Rupp, Antenna selection in polarized multiple input multiple output transmissions with mutual coupling, Integrated Computer-Aided Engineering, 19(3) (2012), 299-312.

[4] J.H. Han and N.H. Myung, Exact and simple calculation of mutual impedance for coplanar-skew dipoles, Electronics Letters 48(8) (2012), 423-425.

[5] J. Richmond and N. Geary, Mutual impedance between coplanar-skew dipoles, IEEE Transactions on Antennas and Propagation 18(3) (1970), 414-416.

[6] J. Zhao, Y. Li and G. Sun, Analysis of antenna mutual coupling in the X-type polarization diversity system, Proceedings of 5th International Conference on Wireless Communications, Networking and Mobile Computing (2009), 1-4.

[7] J. Zhao, Y. Li and G. Sun, The effect of mutual coupling on capacity of 4-element squared antenna array MIMO systems, Proceedings of 5th International Conference on Wireless Communications, Networking and Mobile Computing (2009), 1-4. 